\title{
Efficiency of ultrafast laser-induced demagnetization in $\mathrm{Gd}_{x} \mathrm{Fe}_{100-x-y} \mathrm{Co}_{y}$ alloys
}

\author{
R. Medapalli, ${ }^{*}$ I. Razdolski, M. Savoini, A. R. Khorsand, A. Kirilyuk, A. V. Kimel, ${ }^{\dagger}$ and Th. Rasing \\ Radboud University Nijmegen, Institute for Molecules and Materials, Heyendaalseweg 135, 6525 AJ Nijmegen, The Netherlands
}

A. M. Kalashnikova

Ioffe Physical Technical Institute of the Russian Academy of Sciences, 194021 St. Petersburg, Russia

A. Tsukamoto and A. Itoh

College of Science and Technology, Nihon University, 7-24-1 Funabashi, Chiba, Japan

(Received 2 March 2012; revised manuscript received 1 June 2012; published 29 August 2012)

\begin{abstract}
Laser-induced ultrafast demagnetization in ferrimagnetic $\mathrm{Gd}_{x} \mathrm{Fe}_{100-x-y} \mathrm{Co}_{y}$ thin films was studied experimentally as a function of $\operatorname{Gd}$ concentration $(x=18,22,24,30 \%$, and $y \approx 9-10 \%)$, pump fluence, and sample temperature. The results showed that the conditions for full demagnetization at the ultrafast time scale in $\mathrm{Gd}_{x} \mathrm{Fe}_{100-x-y} \mathrm{Co}_{y}$ thin metal films are easily achieved below the magnetization compensation point $\left(T_{M}\right)$ and, furthermore, when the ratio between $\mathrm{Gd}$ and Fe concentrations is not too large. Consequently, the ultrafast demagnetization strongly depends on the initial temperature of these alloys compared to their $T_{M}$. These results provide further insight into the unconventional ultrafast dynamics of multisublattice metallic magnets.
\end{abstract}

DOI: 10.1103/PhysRevB.86.054442

PACS number(s): 75.50.Gg, 75.50.Kj, 75.78.Jp

\section{INTRODUCTION}

The possibility to manipulate magnetic order with the help of a femtosecond laser pulse has been a subject of intense discussions in modern magnetism ${ }^{1}$ since the seminal observation of subpicosecond laser-induced demagnetization in metals. ${ }^{2}$ The interest in this subject is partly fueled by the fact that ultrafast laser manipulation of magnetic order has a high potential ${ }^{3-7}$ to revolutionize present magnetic recording and information processing technologies. In particular, such an approach is expected to push these technologies into the range of unprecedentedly high operational frequencies above $10 \mathrm{GHz}$, i.e., with writing-reading times less than $100 \mathrm{ps}$.

Most research in ultrafast magnetism is devoted to the investigation of the (sub)picosecond demagnetization in metallic magnetic materials excited by an ultrashort (femtosecond) laser pulse. ${ }^{8-14}$ Although in most cases the demagnetization is not complete, being often at the level of only a few percent, the majority of this research has been focused on understanding the time scale of this demagnetization and on novel channels of angular momentum transfer from the spin system. ${ }^{1}$ At the same time, however, it has been noted that in the case of incomplete demagnetization the rate at which the magnetization drops might have nothing to do with the characteristic interaction time of the spins with other reservoirs of energy and angular momentum (electrons and phonons). Instead, this demagnetization time is mostly determined by the characteristic time of the electron temperature drop. ${ }^{15}$

It is also obvious that, in order to reach an efficient optical control of magnetism in a medium, one should achieve proper conditions for a complete demagnetization or, better, magnetization reversal. ${ }^{16-20}$ Indeed, it was demonstrated recently that if a rare earth (RE)-transition metal (TM) ferrimagnet, having antiferromagnetically coupled nonequivalent RE and TM magnetic sublattices, is brought into a transient state with no net magnetization on a subpicosecond time scale, the subsequent relaxation from this state leads to a deterministic reversal of the initial net magnetization of the medium ${ }^{17}$ if the absorbed energy is great enough. ${ }^{21}$ These and earlier results of all-optical magnetization reversal in $\mathrm{Gd}_{x} \mathrm{Fe}_{100-x-y} \mathrm{Co}_{y}$ alloys $^{18,19}$ logically lead to the question why these RE-TM alloys show such interesting and useful properties. Several parameters of the switching process appear to depend on Gd concentration, which is not well understood so far. ${ }^{20}$ Therefore, understanding how the ultrafast demagnetization of RE-TM alloys depends on Gd concentration, temperature of the sample, and pump fluence is an important and timely issue.

In this article, we present results of time-resolved stroboscopic magneto-optical pump-probe experimental studies of laser-induced ultrafast demagnetization in ferrimagnetic $\mathrm{Gd}_{x} \mathrm{Fe}_{100-x-y} \mathrm{Co}_{y}$ alloys with $\mathrm{Gd}$ concentrations varying between $18 \%$ and $30 \%$. We have investigated the degree of ultrafast demagnetization as a function of sample temperature, laser pump fluence, and concentration of Gd. The degree of demagnetization as a function of laser pulse fluence shows a similar trend in all studied samples. By contrast, variation of the sample temperature affects the ultrafast demagnetization differently in different samples. An analysis of the results clearly shows that the degree of the ultrafast laser-induced demagnetization strongly depends on Gd concentration and in particular on the relative temperature $\left(T_{M}-T\right)$ of the ferrimagnet compared to its magnetization compensation point $T_{M}$. The findings reveal that the ratio between the magnetizations of the $\mathrm{Gd}$ and Fe sublattices plays a crucial role in the process of the laser-induced demagnetization. For instance, the most efficient demagnetization is achieved when the sample temperature is below $T_{M}$ and the magnetizations of the sublattices are comparable. We analyze the revealed trends in light of the latest achievements in the area of ultrafast laser-induced magnetization dynamics of multisublattice magnets. ${ }^{17,19,22}$

This article is structured as follows: In Sec. II we discuss the experimental setup that has been used to study the ultrafast laser-induced magnetization dynamics. Samples and their characterization are discussed in Sec. III. In Sec. IV we 
present the experimental results, followed by a discussion and conclusions in Secs. V and VI, respectively.

\section{EXPERIMENTAL TECHNIQUE}

In order to probe the magnetic state of the metallic alloys, we employed the magneto-optical Faraday effect. The latter is seen as a rotation of the polarization of linearly polarized light over an angle $\theta$ upon propagation through a magnetic sample. The angle of rotation is proportional to the scalar product of the magnetization of the medium $\mathbf{M}$ and the wave vector of light $\mathbf{k}: \theta \sim \mathbf{M}_{Z} \cdot \mathbf{k}$, where $\mathbf{M}_{Z}$ is the projection of $\mathbf{M}$ in the direction of propagation of light. In RE-TM alloys, the magneto-optical susceptibility in (the visible) and near-IR regime is defined by the TM ions. Thus, $\mathbf{M}$ stands for the Fe magnetization.

In order to study the laser-induced magnetization dynamics, a time-resolved stroboscopic magneto-optical pumpprobe technique was employed. Depending on the magnetic anisotropy of the sample, two different configurations of the experimental setup were used. For the study of samples with out-of-plane magnetic anisotropy, the probe beam was set at normal incidence. Such a configuration was chosen in order to maximize the magneto-optical signal from the medium. For the study of the samples with in-plane magnetic anisotropy, the angle of incidence of the probe beam was set to about $60^{\circ}$ in order to increase the $\mathbf{k} \cdot \mathbf{M}_{\mathbf{Z}}$ product. As shown in Fig. 1(a), an external magnetic field $H$ generated by an electromagnet was applied at an angle of $60^{\circ}$ to the sample plane for out-of-plane anisotropy samples, while for in-plane anisotropy samples, $H$ was at an angle of $20^{\circ}$. The angle of incidence for the pump beam was $60^{\circ}$ in both cases.
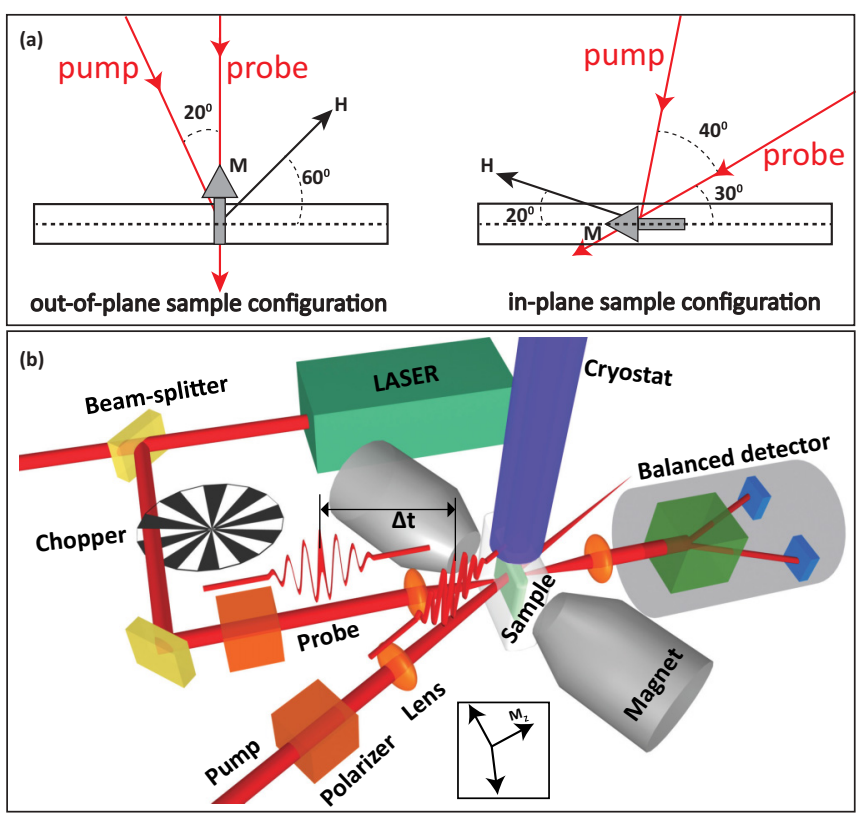

FIG. 1. (Color online) (a) Geometry of the experiments for samples with out-of-plane anisotropy (left) and for samples with in-plane anisotropy (right). (b) Sketch of the time-resolved pumpprobe experimental setup. After every pump pulse, the delayed probe pulse monitors the pump-induced changes in the magnetization of the sample. A balanced detection scheme, consisting of a Wollaston prism and two photodiodes, senses the probe polarization.
The corresponding experimental setup is shown in Fig. 1(b). A Ti:sapphire laser system in combination with an amplifier was used to generate 100 -fs laser pulses with a central photon energy of $1.54 \mathrm{eV}$ and a repetition rate of $1 \mathrm{kHz}$. Linearly polarized pump and probe beams were focused on the samples into spots of around 200 and $100 \mu \mathrm{m}$ in diameter, respectively. The intensity of the pump beam was at least 100 times larger than that of the probe. The delay between the two pulses was controlled by a retroreflector on a motorized translation stage in the pump beam path. After being transmitted through the sample the probe radiation was collected with a balanced detectors scheme [Fig. 1(b)]. In the measurements we used an extra modulation of the probe beam with a chopper operating at $500 \mathrm{~Hz}$ and synchronized with the $1-\mathrm{kHz}$ pulse sequence from the laser amplifier. The difference signal from the photodiodes was first sent to a boxcar integrator triggered by a 1-kHz signal from the laser. The output of the boxcar integrator was further analyzed by a lock-in amplifier which used the 500-Hz signal from the chopper as a reference and then recalculated into the rotation of the probe polarization plane $\theta$. Such a configuration allowed us to detect the polarization rotation $\theta$ while avoiding the effects of scattered light from the pump beam. Measuring the signal from the lock-in amplifier as a function of position of the delay line, we were able to record the temporal behavior of the magneto-optical signal in a laser-excited sample. The pump-probe measurements have been performed for samples with magnetization saturated and oriented in the direction of the easy axis of the magnetic anisotropy. For this we applied a magnetic field slightly above the coercive field $H_{C}$ of the corresponding sample. In order to reduce the influence of possible artifacts irrelevant to the dynamics of the Faraday rotation, we conducted the measurements of the rotation $\theta$ at two opposite directions of applied magnetic fields and took the difference between the measured signals to obtain $\theta_{F}=[\theta(+H)-\theta(-H)] / 2$.

With an optical cold-finger helium flow cryostat, the sample temperature was varied in the range from 10 to $350 \mathrm{~K}$. Note that due to the 1-kHz high-power laser excitation and a nonideal heat transfer from the laser-excited spot, the temperature of the sample was higher than the temperature of the sample holder in the cryostat. This temperature increase was calibrated by comparing absolute values of the static Faraday rotation when the pump beam was present and blocked. Typically, the laser-induced increase of the average sample temperature was below $60 \mathrm{~K}$. This offset heating has been taken into account in all the measurements shown in the following sections.

\section{SAMPLES AND THEIR CHARACTERIZATION}

The ferrimagnetic amorphous rare earth-transition metal (RE-TM) alloys are well known for their applications in magneto-optical storage media. ${ }^{23}$ The ferrimagnetic properties of GdFeCo alloys are essentially determined by the fact that the $\mathrm{Gd}$ spin moments ( $4 f$ and $5 d$ ) are aligned oppositely to the spin moments of $\mathrm{Fe}(3 d)$ and Co. ${ }^{24}$ Because of different temperature dependencies of the magnetization of the sublattices, these alloys possess a magnetization compensation temperature $T_{M}$ in a certain range of Gd concentrations, where the magnetizations of the two sublattices exactly compensate each other. 


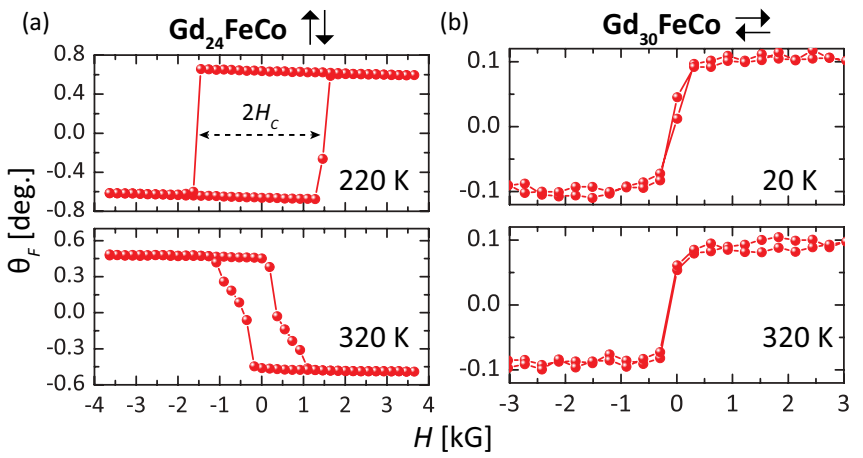

FIG. 2. (Color online) Static Faraday rotation $\theta_{F}$ as a function of magnetic field $H$ in $\mathrm{Gd}_{24} \mathrm{FeCo}$ (out-of-plane anisotropy) and in $\mathrm{Gd}_{30} \mathrm{FeCo}$ (in-plane anisotropy). (a) The measurements for $\mathrm{Gd}_{24} \mathrm{FeCo}$ at $220 \mathrm{~K}$ (top) and $320 \mathrm{~K}$ (bottom). Note the reversal of the hysteresis loop after crossing the compensation point $\left(T_{M}=270 \mathrm{~K}\right)$. (b) The measurements for $\mathrm{Gd}_{30} \mathrm{FeCo}$ at $20 \mathrm{~K}$ (top) and $320 \mathrm{~K}$ (bottom).

The samples studied are thin multilayer films of AlTi (10 $\mathrm{nm}) / \mathrm{SiN}(5 \mathrm{~nm}) / \mathrm{Gd}_{x} \mathrm{Fe}_{100-x-y} \mathrm{Co}_{y}(20 \mathrm{~nm}) / \mathrm{SiN}(60 \mathrm{~nm})$, grown by magnetron sputtering on glass substrates. The AlTi layer serves as a heat sink, and the bottom SiN layer, which is $5 \mathrm{~nm}$ thick, works as a buffer layer while the top one, which is $60 \mathrm{~nm}$ thick, works as a capping layer and antireflection coating. The magnetic properties of $\mathrm{GdFeCo}$, such as coercive field $\left(H_{C}\right)$, saturation magnetization $\left(M_{S}\right)$, magnetic anisotropy, and magnetization compensation temperature $\left(T_{M}\right)$, and also structural properties like crystallinity or amorphousness, all depend on Gd concentration $x$ (in percent). ${ }^{25,26}$

Magneto-optical studies using 800-nm light showed that $\mathrm{Gd}_{18} \mathrm{Fe}_{71.8} \mathrm{Co}_{10.2}$ and $\mathrm{Gd}_{30} \mathrm{Fe}_{61.3} \mathrm{Co}_{8.7}$ films have inplane magnetic anisotropy while $\mathrm{Gd}_{22} \mathrm{Fe}_{68.2} \mathrm{Co}_{9.8}$ and $\mathrm{Gd}_{24} \mathrm{Fe}_{66.5} \mathrm{Co}_{9.5}$ have out-of-plane anisotropy. Figure 2(a) shows the hysteresis loops measured for $\mathrm{Gd}_{24} \mathrm{Fe}_{66.5} \mathrm{Co}_{9.5}$ films at two different temperatures, 220 and $320 \mathrm{~K}$. Since the magneto-optical susceptibility in the spectral range of the probe pulse $(800 \mathrm{~nm})$ is defined by the Fe sublattice, the reversal of the hysteresis loop indicates crossing of the compensation point $T_{M}$. In Fig. 2(b), we showed the hysteresis loops for an in-plane-anisotropy sample $\left(\mathrm{Gd}_{30} \mathrm{FeCo}\right)$ measured at $20 \mathrm{~K}$ and at $320 \mathrm{~K}$. As mentioned before, two different configurations were used depending on the magnetic anisotropy of the sample. The shape of the measured hysteresis loops in both configurations confirmed that the applied magnetic field was strong enough to saturate the magnetization along the easy axis of the samples.

Figure 3(a) shows the temperature dependence of $H_{C}$ extracted from the hysteresis loops, for the samples with different $\mathrm{Gd}$ concentrations: $x=18,22$, and $24 \%$. The divergence of $H_{C}$ at $50 \mathrm{~K}$ and at $270 \mathrm{~K}$ represents the $T_{M}$ of $\mathrm{Gd}_{22} \mathrm{Fe}_{68.2} \mathrm{Co}_{9.8}$ and $\mathrm{Gd}_{24} \mathrm{Fe}_{66.5} \mathrm{Co}_{9.5}$, respectively. In the case of $\mathrm{Gd}_{18} \mathrm{Fe}_{71.8} \mathrm{Co}_{10.2}$ and $\mathrm{Gd}_{30} \mathrm{Fe}_{61.3} \mathrm{Co}_{8.7}$, no $T_{M}$ within the temperature range $10<T<350 \mathrm{~K}$ has been observed. Therefore, we conclude that, in $\mathrm{Gd}_{x} \mathrm{Fe}_{100-x-y} \mathrm{Co}_{y}$, $T_{M}$ increases linearly as a function of $x$ as predicted by Heiman et al. ${ }^{27}$ In $\mathrm{Gd}_{18} \mathrm{Fe}_{71.8} \mathrm{Co}_{10.2}$, the magnetization of $\mathrm{Fe}$ is larger than that of $\mathrm{Gd}$ in the aforementioned temperature range and vice versa in $\mathrm{Gd}_{30} \mathrm{Fe}_{61.3} \mathrm{Co}_{8.7}$. Note that the magneto-optical Faraday rotation, which defines the height of the measured

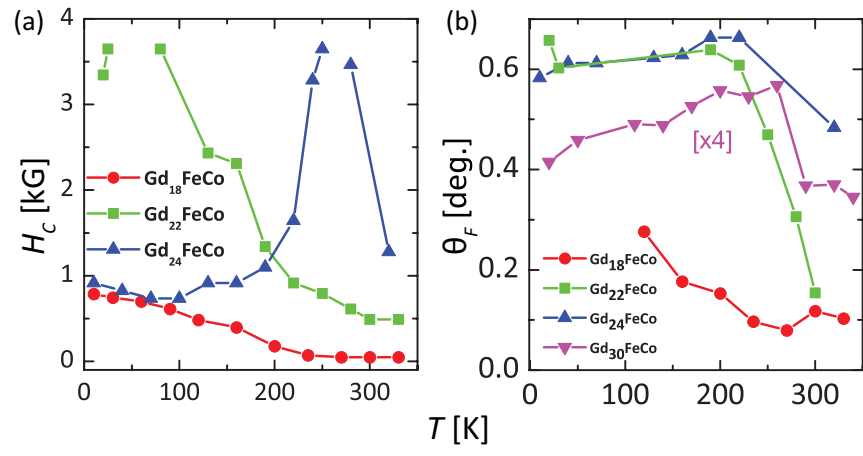

FIG. 3. (Color online) (a) The coercive field $H_{C}$ vs temperature $T$ for three different Gd concentrations ( $x=18,22$, and 24\%). The divergence in the coercivity indicates the magnetization compensation points. Note that $\mathrm{Gd}_{18} \mathrm{FeCo}$ does not have a compensation point within the measured temperature range. In the case of $\mathrm{Gd}_{30} \mathrm{FeCo}$ (not shown), the $H_{C}$ is below $100 \mathrm{G}$ and is independent of sample temperature. (b) The Faraday rotation $\theta_{F}$ vs temperature $T$ curves for four different $\mathrm{Gd}$ concentrations ( $x=18,22,24$, and 30\%). Note that the Faraday rotation decreases with an increase of temperature in all the samples.

hysteresis loop, is also a function of Gd concentration [see Fig. 3(b)]. At room temperature (RT) the Faraday rotation measured in $\mathrm{Gd}_{18} \mathrm{Fe}_{71.8} \mathrm{Co}_{10.2}, \mathrm{Gd}_{22} \mathrm{Fe}_{68.2} \mathrm{Co}_{9.8}, \mathrm{Gd}_{24} \mathrm{Fe}_{66.5} \mathrm{Co}_{9.5}$, and $\mathrm{Gd}_{30} \mathrm{Fe}_{61.3} \mathrm{Co}_{8.7}$ in magnetic fields just above $H_{C}$ reached $0.1^{\circ}, 0.4^{\circ}, 0.5^{\circ}$, and $0.1^{\circ}$, respectively. In fact, in this range of compositions $\mathrm{Fe}$ concentration changes by $12 \%$, i.e., $\mathrm{Fe}$ magnetization values are close in all the samples. The observed difference in $\theta_{F}$ is related to the different magneto-optical susceptibilities caused by different Gd concentrations. Since the percentage of Co in these samples does not vary significantly, in the following we omit the subscripts indicating the concentrations of $\mathrm{Fe}$ and $\mathrm{Co}$. The samples are referred to as $\mathrm{Gd}_{18} \mathrm{FeCo}$, $\mathrm{Gd}_{22} \mathrm{FeCo}, \mathrm{Gd}_{24} \mathrm{FeCo}$, and $\mathrm{Gd}_{30} \mathrm{FeCo}$, respectively.

\section{EXPERIMENTAL RESULTS AND DISCUSSION}

\section{A. Ultrafast laser-induced magnetization dynamics in GdFeCo alloys}

Figure 4(a) shows the temporal evolution of the polarization rotation $\theta$ in $\mathrm{Gd}_{18} \mathrm{FeCo}$ excited at time 0 fs by an intense 100 -fs laser pulse for two opposite field directions. The measured curves have been normalized to the signal at negative time delay $\theta(-1 \mathrm{ps})$. The photoinduced change of transmission through our samples was observed to be well below $6 \%$. At the same time, $\theta / \theta(-1 \mathrm{ps})$ experiences much larger changes. Therefore, we can safely assign the dynamics of the difference between $\theta$ measured at the two magnetic fields to the dynamics of the Faraday rotation $\theta_{F}=[\theta(+H)-\theta(-H)] / 2$. Figure 4(b) shows the dynamics of the Faraday rotation normalized to the rotation at negative time delay $\theta_{F} / \theta_{F}(-1$ ps). It is seen that, within $300 \mathrm{fs}$ after the laser excitation, the magneto-optical signal significantly reduces and stays at the reduced level longer than $5 \mathrm{ps}$. The following recovery is slow and is observed on a nanosecond time scale. We note that at a 2 ps time delay the processes of both optical decoherence and thermalization of the electronic system are expected to be completed. ${ }^{13}$ Therefore, we confidently ascribe the 


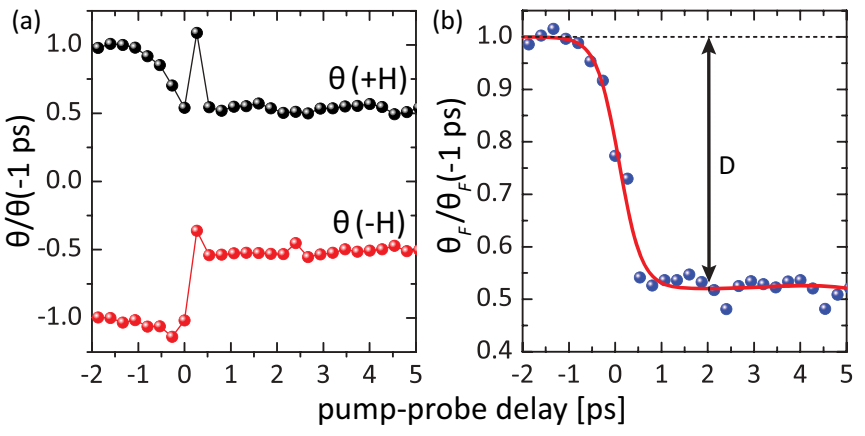

FIG. 4. (Color online) (a) Dynamics of polarization rotation in $\mathrm{Gd}_{18} \mathrm{FeCo}$ induced by ultrashort laser pulses with a fluence of $13.4 \mathrm{~mJ} / \mathrm{cm}^{2}$ measured at room temperature for two opposite directions of the external magnetic field $H=2.4 \mathrm{kG}$. (b) Laserinduced dynamics of the Faraday rotation $\theta_{F}$. Here $D$ indicates the degree of demagnetization. Solid line is the fit to the experimental data, used to extract $D$.

magneto-optical signal at time delays longer than 2 ps to the dynamics of the magnetization of the Fe sublattice: $\theta_{F} / \theta_{F}(-1 \mathrm{ps})=M_{Z} / M_{Z}(-1 \mathrm{ps})$. The reduction of the magnetization within 2 ps is considered the amount of ultrafast demagnetization or degree of demagnetization, $D$, and is defined in Eq. (1):

$$
D=\frac{\theta_{F}(-1 \mathrm{ps})-\theta_{F}(+2 \mathrm{ps})}{\theta_{F}(-1 \mathrm{ps})} .
$$

One may argue that the observed changes of the magnetooptical signal can be due to a reorientation of magnetization over a large angle. It is indeed known that an ultrafast excitation of a metal can change its effective magnetic anisotropy field and trigger magnetization reorientation. ${ }^{4,28}$ We would like to note, however, that the measured transient signal is characterized by a subpicosecond drop followed by a slow recovery on a nanosecond time scale with no sign of oscillations. If one assumes that the subpicosecond drop is due to a tilt of the magnetization, it would mean that the magnetic system is characterized by very high magnetic resonance frequencies (with precession periods in the subpicosecond time domain) and an extremely high damping (it should be high enough that a triggered precession is damped over a quarter of the period). However, such high values are not confirmed either by experimental $^{18}$ or theoretical studies ${ }^{29}$ of magnetic resonance in GdFeCo alloys. Moreover, the observed values of the demagnetization are at least as large as $50 \%$. If such a change is caused by a reorientation of the magnetization, it would mean that the magnetization rotates over at least $60^{\circ}$. However, it seems that there is no reason for such a huge change of the equilibrium orientation. A subpicosecond magnetization reorientation would mean that effective magnetic anisotropy changes at an even faster time scale. Such a fast change cannot be due to a change of magnetocrystalline anisotropy ${ }^{28}$ and must originate from ultrafast demagnetization of the material. Saturation magnetization $\left(M_{S}\right)$ in the studied compounds is of the order of $50 \mathrm{G}$ or even less. ${ }^{25} 4 \pi M_{S}$ is much smaller than the applied magnetic field in our experiment (2.5 kG). Therefore, demagnetization will not be able to cause a sufficient change of the equilibrium orientation of
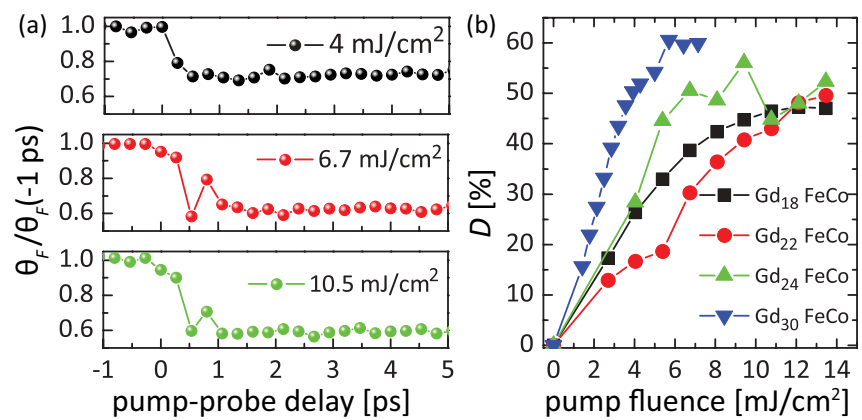

FIG. 5. (Color online) (a) Time dependence of ultrafast demagnetization in $\mathrm{Gd}_{18} \mathrm{FeCo}$ at room temperature at three different pump fluences, 4.0, 6.7, and $10.5 \mathrm{~mJ} / \mathrm{cm}^{2}$, respectively. (b) Corresponding degree of demagnetization $D$ as a function of the pump pulse fluence measured at RT for various samples.

the magnetic moment. Hence, we are strongly convinced that the observed ultrafast demagnetization is purely a reduction in magnetization that still stays along the easy axis.

\section{B. Fluence dependence of ultrafast demagnetization}

Figure 5(a) shows the dynamics of the ultrafast demagnetization in $\mathrm{Gd}_{18} \mathrm{FeCo}$ at three different pump fluences, 4.0, 6.7 , and $10.5 \mathrm{~mJ} / \mathrm{cm}^{2}$, at room temperature. It is seen that $D$ increases with an increase of pump fluence. Performing the measurements for a wide range of fluences, we found that $D$ increases up to $10 \mathrm{~mJ} / \mathrm{cm}^{2}$ and saturates for higher fluences at the level of nearly $40-60 \%$. The fluence dependence of $D$ is given in Fig. 5(b).

Similar experiments at room temperature have also been carried out on $\mathrm{Gd}_{22} \mathrm{FeCo}, \mathrm{Gd}_{24} \mathrm{FeCo}$, and $\mathrm{Gd}_{30} \mathrm{FeCo}$ samples [see Fig. 5(b)]. A general trend holds for all samples; i.e., demagnetization occurs within the first few hundred femtoseconds. In all the samples we have also observed an increase in $D$ followed by saturation upon increasing the pump fluence. Slightly above the saturation pump fluence, optical excitation results in irreversible changes in all the samples. It should be emphasized that this damage threshold is obtained for the case of excitation with a $1-\mathrm{kHz}$ sequence of the laser pulses when the time between two successive pump pulses is not sufficient for heat dissipation. By decreasing the repetition rate, one should be able to increase the damage threshold. Consequently, in experiments with a lower repetition rate, a higher degree of the demagnetization can be expected.

\section{Temperature dependence of ultrafast demagnetization}

Figure 6(a) shows the dynamics of the magneto-optical signal $\theta_{F} / \theta_{F}(-1 \mathrm{ps})$ measured in $\mathrm{Gd}_{18} \mathrm{FeCo}$ for a fixed pump fluence of $10.5 \mathrm{~mJ} / \mathrm{cm}^{2}$ at three different temperatures, 40 , 265 , and $330 \mathrm{~K}$. It can be seen that the dynamics of the magneto-optical signal shows a weak dependence on the sample temperature. Detailed measurements in the temperature range 40-360 $\mathrm{K}$ showed that the degree of demagnetization $D$ increases slightly from $35 \%$ to $45 \%$ upon a temperature increase within this range [see Fig. 6(b)].

The situation changes strongly when the concentration of Gd gets a little higher. Figure 7(a) shows the dynamics of 

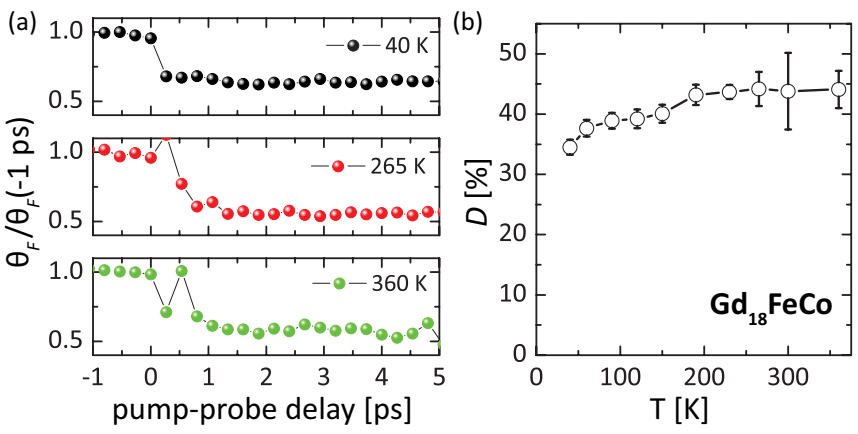

FIG. 6. (Color online) (a) Time dependence of ultrafast demagnetization in $\mathrm{Gd}_{18} \mathrm{FeCo}$ at three different temperatures, 40, 265, and $360 \mathrm{~K}$ (top to bottom), is shown at a fixed pump fluence of $10.5 \mathrm{~mJ} / \mathrm{cm}^{2}$. (b) Extracted degree of ultrafast demagnetization $D$ as a function of $T$.

the magneto-optical Faraday effect in the $\mathrm{Gd}_{22} \mathrm{FeCo}$ sample at three different temperatures, 70, 130, and $370 \mathrm{~K}$. As one can see in Fig. 7(b), the degree of demagnetization $D$ appears to be very sensitive for the sample temperature and depends on the latter in a nonmonotonous way. A heating of the sample from 70 to $140 \mathrm{~K}$ results in a drop of $D$ from $70 \%$ to $30 \%$. Further heating of the alloy leads to a partial recovery of the demagnetization efficiency so that at room temperature $D$ is comparable to the one in $\mathrm{Gd}_{18} \mathrm{FeCo}$.

Further increase in the $\mathrm{Gd}$ concentration results in even more dramatic changes of the laser-induced dynamics in the alloys. Figure 8(a) shows the dynamics of the magneto-optical Faraday rotation measured in $\mathrm{Gd}_{24} \mathrm{FeCo}$ at different temperatures at a pump fluence of $10.7 \mathrm{~mJ} / \mathrm{cm}^{2}$. If the temperature of the sample is below the compensation point, a pump pulse of fluence $10.7 \mathrm{~mJ} / \mathrm{cm}^{2}$ causes $100 \%$ demagnetization of the alloy [see Fig. 8(b)]. However, just above the compensation point the same fluence causes just $50 \%$ demagnetization. At room temperature the degree of the demagnetization of $\mathrm{Gd}_{24} \mathrm{FeCo}$ is comparable to the one in $\mathrm{Gd}_{18} \mathrm{FeCo}$ and in $\mathrm{Gd}_{22} \mathrm{FeCo}$. Therefore, the results obtained for $\mathrm{Gd}_{18} \mathrm{FeCo}$, $\mathrm{Gd}_{22} \mathrm{FeCo}$, and $\mathrm{Gd}_{24} \mathrm{FeCo}$ alloys reveal that the presence of the magnetization compensation point plays an important role in the process of the subpicosecond demagnetization. Indeed, in $\mathrm{Gd}_{18} \mathrm{FeCo}$, which does not possess a compensation point, no pronounced temperature dependence of $D$ is observed. In
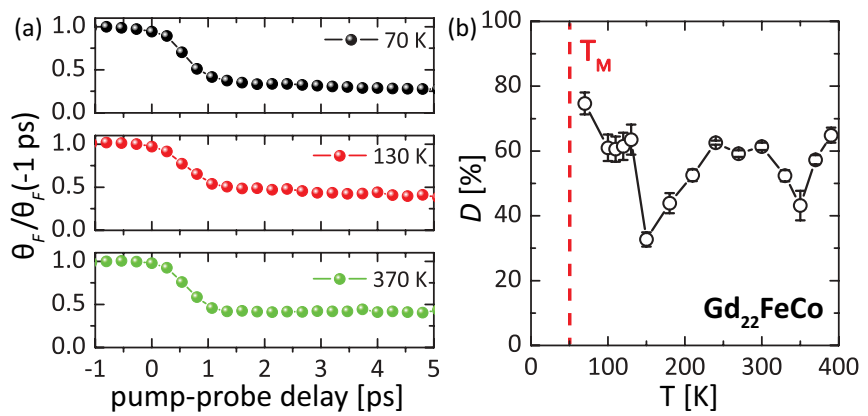

FIG. 7. (Color online) (a) Time dependence of ultrafast demagnetization in $\mathrm{Gd}_{22} \mathrm{FeCo}$ at three different temperatures, 70, 130, and 370 $\mathrm{K}$, is shown at a fixed pump fluence of $10.5 \mathrm{~mJ} / \mathrm{cm}^{2}$. (b) Extracted degree of ultrafast demagnetization $D$ as a function of $T$.
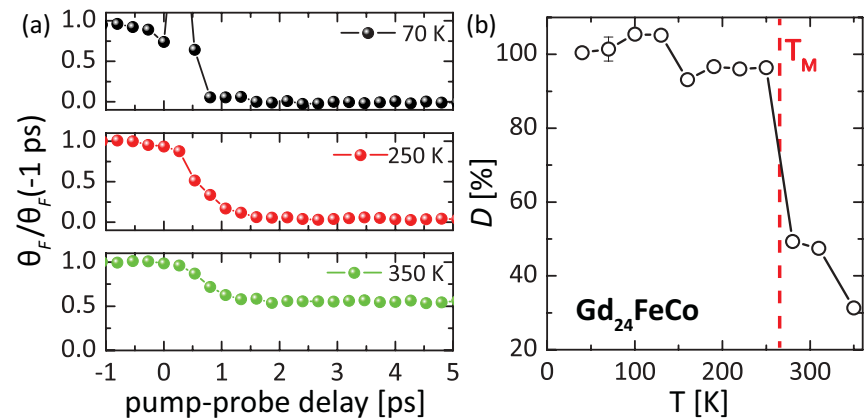

FIG. 8. (Color online) (a) Time dependence of ultrafast demagnetization in $\mathrm{Gd}_{24} \mathrm{FeCo}$ at three different temperatures, 70, 250, and $350 \mathrm{~K}$, is shown at fixed fluence of $10.7 \mathrm{~mJ} / \mathrm{cm}^{2}$. (b) Extracted degree of ultrafast demagnetization $D$ as a function of $T$.

contrast, in $\mathrm{Gd}_{22} \mathrm{FeCo}$ the compensation point lies just below the studied temperature range and it is seen that the efficiency of the demagnetization for a given laser pulse fluence increases upon cooling the sample towards $T_{M}$. This effect becomes even more pronounced in $\mathrm{Gd}_{24} \mathrm{FeCo}$, where the magnetization compensation point lies just in the middle of the studied range of temperatures. The data obtained for this alloy show that heating farther away from the compensation point decreases the degree of demagnetization. Furthermore, laser excitation demagnetizes the alloys most efficiently at temperatures below the compensation point.

Finally, we studied the temperature dependence of the demagnetization in the $\mathrm{Gd}_{30}$ FeCo sample in which no magnetization compensation point is observed and the magnetization of the Gd sublattice is always larger than the one of Fe. Figure 9 (a) shows the dynamics of the Faraday rotation $\theta_{F} / \theta_{F}(-1 \mathrm{ps})$, measured in $\mathrm{Gd}_{30} \mathrm{FeCo}$ at three different temperatures, 20, 140 , and $320 \mathrm{~K}$. In the range between 50 and $300 \mathrm{~K}, D$ is $40 \%$ and almost does not depend on the temperature. An increase of $D$ from $40 \%$ to $70 \%$ is observed when the sample temperature is increased from 300 to $350 \mathrm{~K}$ [see Fig. 9(b)]. Hence, again a slight change of Gd concentration resulted in a dramatic change in the degree of demagnetization $D$. All these experimental observations reveal that the degree of ultrafast laser-induced demagnetization is strongly dependent on the presence of a compensation temperature $T_{M}$ and the relative temperature of the sample with respect to $T_{M}$.
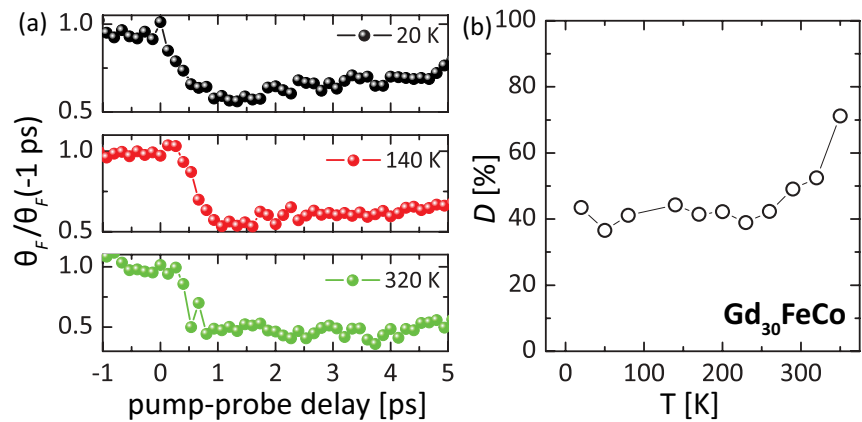

FIG. 9. (Color online) (a) Time dependence of ultrafast demagnetization in $\mathrm{Gd}_{30} \mathrm{FeCo}$ at three different temperatures, 20, 140, and 320 $\mathrm{K}$, is shown at a fixed pump fluence of $10.5 \mathrm{~mJ} / \mathrm{cm}^{2}$. (b) Extracted degree of ultrafast demagnetization $D$ as a function of $T$. 


\section{DISCUSSION}

As mentioned above, in the described experiments, the magneto-optical signal is dominated by the response of the Fe sublattice. In the studied alloys Fe constitutes $60-72 \%$ per chemical formula unit, and, consequently, the absolute value of the magnetization of the Fe sublattice is approximately the same in all the studied alloys. However, it appears that small changes in the compositions or the temperature of the sample lead to dramatic changes in the degree of the ultrafast demagnetization of the Fe sublattice.

Starting from the very first study of laser-induced demagnetization of magnetic metals, the transient laser-induced processes have been described in terms of a three-temperature model, where spins, free electrons, and the lattice played the roles of reservoirs of energy and angular momentum. ${ }^{30}$ In these models an ultrafast laser excitation resulted in a rapid absorption of energy by the electron system, followed by a redistribution of energy from the electrons to the spins and lattice, accompanied by a flow of angular momentum from the spin system to the two other reservoirs. So far, most of the models of laser-induced magnetization dynamics have treated the spins as one reservoir, even in cases where the system consists of two or more spin sublattices. Only very recent theoretical treatments ${ }^{17,22}$ and time-resolved and element-specific experiments ${ }^{19}$ on GdFeCo alloys showed that such an approach has a serious shortcoming. It was discovered that ultrafast laser excitation of $\mathrm{GdFeCo}$ causes ultrafast collapse and subsequent reversal of the $\mathrm{Gd}$ and Fe sublattice magnetizations over distinctly different time scales. ${ }^{19}$ This clearly showed that the spin system in such a complex magnetic medium should not be considered as a single reservoir. Instead, transfer of angular momentum between these spin reservoirs should be considered. ${ }^{17,22}$

In the light of these findings it becomes clear that our experimental results reveal only the degree of ultrafast demagnetization of the Fe sublattice, while demagnetization of the Gd sublattice is expected to happen over a longer time scale and is not monitored in our experiments. In $\mathrm{Gd}_{24} \mathrm{FeCo}$ below $T_{M}(=270 \mathrm{~K})$, the Gd sublattice has a larger magnetization than the Fe sublattice. Consequently, Gd is an effective reservoir of angular momentum that, in particular, allows one to reach $100 \%$ ultrafast demagnetization of the Fe sublattice which was seen in our experiments. Above $T_{M}$ the Fe sublattice has a magnetic moment larger than the Gd one and full demagnetization of the former would mean reversal of the net magnetization. Obviously, demagnetization above $T_{M}$ is more difficult than below. As a result, laser pulses of the same fluence cause $100 \%$ demagnetization of $\mathrm{Fe}$ in $\mathrm{Gd}_{24} \mathrm{FeCo}$ below the compensation point and just a partial demagnetization above $T_{M}$.

The results obtained in $\mathrm{Gd}_{22} \mathrm{FeCo}$ in the range up to $150 \mathrm{~K}$ and in $\mathrm{Gd}_{18} \mathrm{FeCo}$ can also be understood along the same line. In both cases $\mathrm{Gd}$ constitutes an even smaller reservoir of angular momentum than in $\mathrm{Gd}_{24} \mathrm{FeCo}$. However, experimental results on ultrafast demagnetization of the Fe sublattice in $\mathrm{Gd}_{30} \mathrm{FeCo}$ reveal that if the net magnetization of $\mathrm{Gd}$ is getting larger than that of Fe, the efficiency of the demagnetization decreases despite an increasing capacity of the Gd reservoir. A qualitative theoretical analysis ${ }^{22}$ allows us to suggest that this can be due to a reduction of the Fe-Fe exchange interaction. Such a reduction would result in a slowdown of the process of ultrafast demagnetization. Since the latter can only occur during a limited period of time between the moment of excitation of the metal until the free-electron gas cools down below the Curie temperature, this would show up in our experiments as a decrease in the degree of demagnetization. We would like to stress, however, that the suggested explanation is very qualitative. An interesting behavior has been observed in the temperature dependencies obtained for $\mathrm{Gd}_{22} \mathrm{FeCo}$ and $\mathrm{Gd}_{30} \mathrm{FeCo}$ in the ranges above 150 and $300 \mathrm{~K}$, respectively. In particular, an increase of the temperature of $\mathrm{Gd}_{22} \mathrm{FeCo}$ above $150 \mathrm{~K}$ results in a nonmonotonic behavior of the degree of the ultrafast demagnetization. An increase of the temperature of $\mathrm{Gd}_{30} \mathrm{FeCo}$ above $300 \mathrm{~K}$ leads to a substantial change of the degree of demagnetization as well. We must admit that we are unable to clarify the origin of these features yet. However, it must be mentioned that to the best of our knowledge even the fact that the most efficient ultrafast demagnetization of the Fe sublattice in the GdFeCo alloy is observed when the magnetizations of $\mathrm{Gd}$ and $\mathrm{FeCo}$ are close to one another cannot be unambiguously predicted by any of the existing theories. Therefore, we believe that our experimental findings will stimulate further theoretical analysis of the problem of ultrafast laser-induced demagnetization in multisublattice magnets.

\section{CONCLUSIONS}

With the aid of time-resolved pump-probe experiments, we have examined the ultrafast demagnetization efficiency in various $\mathrm{Gd}_{x} \mathrm{Fe}_{100-x-y} \mathrm{Co}_{y}$ alloys as a function of $\mathrm{Gd}$ concentration $(x=18,22,24$, and $30 \%)$ and sample temperature. The difference in demagnetization dynamics in $\mathrm{Gd}_{24} \mathrm{FeCo}$ above and below $T_{M}$ shows that the ultrafast demagnetization is more efficient below $T_{M}$; i.e., the initial temperature of the sample plays an important role. A comparison of the degree of demagnetization in $\mathrm{Gd}_{18} \mathrm{FeCo}$ and $\mathrm{Gd}_{30} \mathrm{FeCo}$ with that of $\mathrm{Gd}_{24} \mathrm{FeCo}$ shows that the more effective demagnetization takes place when the magnetizations of the individual atomic sublattices ( $\mathrm{Fe}$ and $\mathrm{Gd}$ ) are approximately equal. Finally, we would like to note that since achieving the conditions for $100 \%$ demagnetization is of crucial importance for the realization of all-optical switching via a strongly nonequilibrium state, ${ }^{16,17,20}$ our results clearly show that the all-optical switching is most easily observed in ferrimagnets with a magnetic compensation point. All-optical switching requires less laser pulse fluence when the sample is below the compensation temperature. Finally, our experiments clearly show that heating a ferrimagnetic material farther away from the point of magnetic compensation makes laser-induced demagnetization and magnetization reversal increasingly difficult.

\section{ACKNOWLEDGMENTS}

The authors would like to thank A. van Rooij, T. Toonen, and A. van Etteger for technical support. We also would like to thank F. Atoneche for help in the measurements and J. Mentink for fruitful discussions. This work was partially supported by de Nederlandse Organisatie voor Wetenschappelijk Onderzoek (NWO), NanoSci-E+ program, Foundation for Fundamental 
Research (FOM) and the Technology Foundation (STW), the Russian Foundation for Basic Research (RFBR) Grant No. 10-02-01008-a, the European Union's Seventh Framework Programme (FP7/2007-2013) Grants No. NMP3-SL-2008214469 (UltraMagnetron) and No. 214810 (FANTOMAS), as well as the European Research Council under the European Union's Seventh Framework Programme (FP7/20072013)/ERC Grant No. 257280 (Femtomagnetism). The authors would also like to thank Nihon University Strategic Projects for Academic Research. *r.medapalli@science.ru.nl

†a.kimel@science.ru.nl

${ }^{1}$ A. Kirilyuk, A. V. Kimel, and Th. Rasing, Rev. Mod. Phys. 82, 2731 (2010).

${ }^{2}$ E. Beaurepaire, J. C. Merle, A. Daunois, and J. Y. Bigot, Phys. Rev. Lett. 76, 4250 (1996).

${ }^{3}$ J. Hohlfeld, T. Gerrits, M. Bilderbeek, T. Rasing, H. Awano, and N. Ohta, Phys. Rev. B 65, 012413 (2001).

${ }^{4}$ M. van Kampen, C. Jozsa, J. T. Kohlhepp, P. LeClair, L. Lagae, W. J. M. de Jonge, and B. Koopmans, Phys. Rev. Lett. 88, 227201 (2002).

${ }^{5}$ A. V. Kimel, A. Kirilyuk, A. Tsvetkov, R. V. Pisarev, and T. Rasing, Nature (London) 429, 850 (2004).

${ }^{6}$ G. Ju, J. Hohlfeld, B. Bergman, R. J. M. van de Veerdonk, O. N. Mryasov, J. Y. Kim, X. W. Wu, D. Weller, and B. Koopmans, Phys. Rev. Lett. 93, 197403 (2004).

${ }^{7}$ J. U. Thiele, M. Buess, and C. H. Back, Appl. Phys. Lett. 85, 14 (2004).

${ }^{8}$ H. A. Dürr, C. Stamm, T. Kachel, N. Pontius, R. Mitzner, T. Quast, K. Holldack, S. Khan, C. Lupulescu, E. F. Aziz, W. M. Wietstruk, and W. Eberhardt, IEEE Trans. Magn. 44, 1957 (2008).

${ }^{9}$ E. Carpene, E. Mancini, C. Dallera, M. Brenna, E. Puppin, and S. De Silvestri, Phys. Rev. B 78, 174422 (2008).

${ }^{10}$ M. Krauss, T. Roth, S. Alebrand, D. Steil, M. Cinchetti, M. Aeschlimann, and H. C. Schneider, Phys. Rev. B 80, 180407 (2009).

${ }^{11}$ T. Kampfrath, R. G. Ulbrich, F. Leuenberger, M. Munzenberg, B. Sass, and W. Felsch, Phys. Rev. B 65, 104429 (2002).

${ }^{12}$ G. P. Zhang and W. Hubner, Phys. Rev. Lett. 85, 3025 (2000).

${ }^{13}$ P. M. Oppeneer and A. Liebsch, J. Phys.: Condens. Matter 16, 5519 (2004).

${ }^{14}$ B. Koopmans, G. Malinowski, F. D. Longa, D. Steiauf, M. Fähnle, T. Roth, M. Cinchetti, and M. Aeschlimann, Nature Mater. 9, 259 (2010).

${ }^{15}$ J. Wang, C. Sun, J. Kono, A. Oiwa, H. Munekata, L. Cywinski, and L. J. Sham, Phys. Rev. Lett. 95, 167401 (2005).

${ }^{16}$ K. Vahaplar, A. M. Kalashnikova, A. V. Kimel, D. Hinzke, U. Nowak, R. Chantrell, A. Tsukamoto, A. Itoh, A. Kirilyuk, and T. Rasing, Phys. Rev. Lett. 103, 117201 (2009).
${ }^{17}$ T. A. Ostler, J. Barker, R. F. L. Evans, R. W. Chantrell, U. Atxitia, O. Chubykalo-Fesenko, S. El Moussaoui, L. Le Guyader, E. Mengotti, L. J. Heyderman, F. Nolting, A. Tsukamoto, A. Itoh, D. Afanasiev, B. A. Ivanov, A. M. Kalashnikova, K. Vahaplar, J. Mentink, A. Kirilyuk, and Th. Rasing, Nat. Commun. 3, 666 (2012).

${ }^{18}$ C. D. Stanciu, A. V. Kimel, F. Hansteen, A. Tsukamoto, A. Itoh, A. Kirilyuk, and Th. Rasing, Phys. Rev. B 73, 220402 (2006).

${ }^{19}$ I. Radu, K. Vahaplar, C. Stamm, T. Kachel, N. Pontius, H. A. Dürr, T. A. Ostler, J. Barker, R. F. L. Evans, R. W. Chantrell, A. Tsukamoto, A. Itoh, A. Kirilyuk, Th. Rasing, and A. V. Kimel, Nature (London) 472, 205 (2011).

${ }^{20}$ K. Vahaplar, A. M. Kalashnikova, A. V. Kimel, S. Gerlach, D. Hinzke, U. Nowak, R. Chantrell, A. Tsukamoto, A. Itoh, A. Kirilyuk, and Th. Rasing, Phys. Rev. B 85, 104402 (2012).

${ }^{21}$ A. R. Khorsand, M. Savoini, A. Kirilyuk, A. V. Kimel, A. Tsukamoto, A. Itoh, and Th. Rasing, Phys. Rev. Lett. 108, 127205 (2012).

${ }^{22}$ J. H. Mentink, J. Hellsvik, D. V. Afanasiev, B. A. Ivanov, A. Kirilyuk, A. V. Kimel, O. Eriksson, M. I. Katsnelson, and Th. Rasing, Phys. Rev. Lett. 108, 057202 (2012).

${ }^{23}$ R. M. White, Science 229, 11 (1985).

${ }^{24}$ M. Taborelli, R. Allenspach, G. Boffa, and M. Landolt, Phys. Rev. Lett. 56, 2869 (1986).

${ }^{25}$ Y. Mimura, N. Imamura, T. Kobayashi, A. Okada, and Y. Kushiro, J. Appl. Phys. 49, 1208 (1978).

${ }^{26}$ A. Takenouchi, E. Shichi, T. Kato, S. Tsunashima, S. Uchiyama, and N. Imamura, J. Appl. Phys. 55, 2164 (1984).

${ }^{27}$ N. Heiman, K. Lee, R. I. Potter, and S. Kirkpatrick, J. Appl. Phys. 47, 2634 (1976).

${ }^{28}$ J.-Y. Bigot, M. Vomir, L. H. F. Andrade, and E. Beaurepaire, Chem. Phys. 318, 137 (2005).

${ }^{29}$ F. Schlickeiser, U. Atxitia, S. Wienholdt, D. Hinzke, O. ChubykaloFesenko, and U. Nowak (unpublished).

${ }^{30}$ M. B. Agranat, S. I. Ashitkov, A. B. Granovsky, and G. I. Rukman, Zh. Eksp. Teor. Fiz. 86, 1376 (1984) [Sov. Phys. JETP 59, 804 (1984)]. 